CASO CLÍNICO

\title{
Reporte de caso: Meningioma anaplásico
}

\section{Anaplastic Meningioma: Case Report}

José Sánchez-Peña*, Boris Torres-Cuevas, Maricel Rodríguez-Cheong y América Pérez-Echeverría

Servicio de Radiología e Imágenes Diagnósticas, Hospital Clínico Quirúrgico Docente Hermanos Ameijeiras, La Habana, Cuba

\section{RESUMEN}

El meningioma es un tumor primario del sistema nervioso central, que representa el tumor cerebral más común, aproximadamente un tercio de los tumores intracraneales primarios. El 90\% son benignos y entre el 1 y el 10\% son malignos. Se presenta el caso de una paciente de 59 años, con alteraciones en la conducta y dificultades motoras. Se había realizado diagnóstico de meningioma de convexidad parietotemporal derecha e intervenido con craneotomía osteoclástica frontotemporal derecha, con exéresis del tumor. A los 2 meses se diagnostica meningioma maligno, usando exámenes radiológicos.

Palabras clave: Meningioma anaplásico. Meningioma maligno. Neoplasia.

\section{ABSTRACT}

Meningioma is a primary tumor of the central nervous system which represents the most common brain tumor in about one-third of primary intracranial tumors. $90 \%$ are benign and between 1 and 10\% are malignant. We present the case of a 59-year-old woman, a patient with alterations in behavior and motor difficulties. A meningioma in the right convexity parietal-temporal was diagnosed and a right fronto-temporal osteoclastic craniotomy with excision of the tumor was performed. After 2 months, a malignant meningioma was diagnosed using radiologic tests.

Key words: Anaplastic meningioma. Malignant meningioma. Neoplasia. 


\section{INTRODUCCIÓN}

Los meningiomas son tumores que se originan de las células meningoteliales (aracnoides) en la superficie interna de la duramadre y representan del 13 al 37\% de los tumores intracraneales primarios ${ }^{1}$. El término meningioma fue introducido por Cushing (1992), dada su relación con la histogénesis y las cubiertas meníngeas ${ }^{2}$.

Aproximadamente el $90 \%$ de los casos son benignos (grado I de la Organización Mundial de la salud [OMS]). El meningioma anaplásico o maligno corresponde al grado III de la OMS, su prevalencia oscila entre el 1 y el 2.8\%, y tiene un comportamiento clínico más agresivo ${ }^{1,3}$. En cuanto a su localización, la convexidad y la región parasagital son las más frecuentes ${ }^{4,5}$.

Los meningiomas se presentan más comúnmente en adultos de edad media y avanzada. El riesgo de tener un meningioma se incrementa con la edad en ambos sexos. Tienen mayor incidencia en las mujeres. Con respecto a esto, se han encontrado receptores hormonales para progesterona y estrógenos. Son de causa desconocida, aunque se han reconocido factores de riesgo como la exposición a radiación y padecer neurofibromatosis de tipo $2^{6,7}$.

Pueden ser incidentales, pequeños y de lento crecimiento, o masas extensas y de crecimiento progresivo ${ }^{5}$. No es poco frecuente el diagnóstico tardío, ya cuando el tamaño y extensión son considerables, lo que puede dificultar su clasificación y manejo.

En los meningiomas de grado III, el tratamiento de elección es la cirugía, seguida de radioterapia. En los estudios realizados se reporta una supervivencia global inferior a 2-3 años ${ }^{8}$. Debido a la agresividad del tumor, el control local es deficiente, incluso con un tratamiento agresivo. Se requieren mejores paradigmas de tratamiento ${ }^{9}$, pues no existen guías de tratamiento estándar debido a la ausencia de estudios prospectivos.

Los meningiomas malignos muestran una razón de recurrencia significativamente superior que los de tipo benigno, incluso después del tratamiento con cirugía y radioterapia ${ }^{10}$. En ocasiones se requieren resecciones quirúrgicas múltiples en pacientes con meningioma recurrente, lo cual reduce la posibilidad de curación e incrementa el riesgo de infección intracraneal, entre otras complicaciones postoperatorias $^{11}$.

\section{PRESENTACIÓN DE CASO}

Paciente de sexo femenino, de 59 años de edad, con antecedentes personales de hipertensión arterial, enfisema pulmonar y granulomatosis anular. Refirió alergia a la tetraciclina y hábitos tóxicos de consumo frecuente de alcohol y fumadora de 10 cigarrillos diarios.

\section{Historia de la enfermedad actual}

La paciente es llevada al hospital por sus familiares en febrero de 2019, con un cuadro de 5 meses de evolución, caracterizado por alteraciones del comportamiento, dado por irritabilidad, con déficit motor izquierdo progresivo, dificultad para la marcha, debilidad muscular en los miembros superior e inferior izquierdos y relajación de esfínteres. 
Fue atendida en primera instancia en el municipio de Morón, en la provincia Ciego de Ávila, donde le realizaron estudios de neuroimágenes (tomografía computarizada [TC] craneal) el 3 de diciembre de 2018, en el que se evidenció una lesión expansiva, de convexidad derecha en la región parietotemporal. El día 14 de diciembre de 2018 es intervenida quirúrgicamente, se le realiza una craneotomía osteoclástica frontotemporal derecha, la cual se complicó con sangrado importante, por lo que tuvo que realizarse la contención de la hemorragia. Fue reintervenida por segunda ocasión el día 18 de diciembre de 2018, sin cambios en la condición y evolución clínica, se le realizó exéresis completa del tumor y derivación ventriculoperitoneal, sin complicaciones.

\section{Examen físico}

Peso de $40 \mathrm{~kg}$, talla de $1.55 \mathrm{~cm}$, índice de masa corporal de $16.6 \mathrm{~kg} / \mathrm{m}^{2}$ y desnutrición moderada. Hemiparesia izquierda a predominio braquial, signo de Babinski positivo en el miembro inferior izquierdo y Glasgow 15/15.

\section{Diagnóstico presuntivo}

Meningioma de la convexidad parietotemporal derecha.

\section{Indicaciones}

Ultrasonido (US) abdominal, TC craneal simple y angiotomografia computarizada (angio-TC).

Los resultados de la TC craneal simple (24 de febrero de 2019) describieron una gran masa

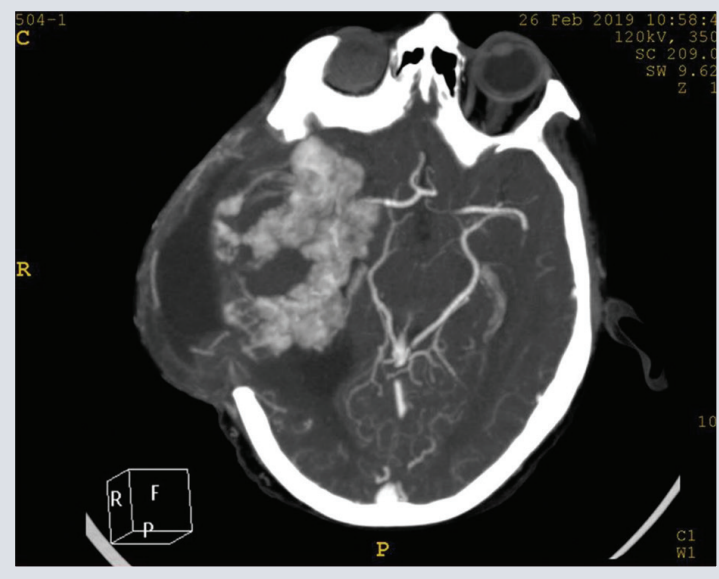

Figura 1. Imagen axial en algoritmo con MIP. Herniación tumoral con compresión sobre territorio de la arteria cerebral media derecha. Nótese los abundantes cuerpos de psamoma. MIP: proyección de máxima intensidad.

heterogénea, predominantemente hiperdensa en la región temporal derecha, que producía un gran efecto de masa, con compresión del sistema ventricular, parte de la misma herniada a través de la craneotomía, así como parte del lóbulo, y presencia de clips quirúrgico. El estudio sugiere diagnóstico de meningioma pterional (anaplásico o maligno).

El resultado de la angio-TC (26 de febrero de 2019) describe herniación del tejido tumoral residual y masa encefálica a través de la puerta de entrada quirúrgica (Fig. 1). Se comprobó una notable vascularización de la lesión, con aferencia de manera significativa a través del grupo medio de la carótida externa derecha, en especial de las arterias temporal superficial y meníngea media (Fig. 2). Por vía de la carótida interna, se observó la participación de la arteria cerebral media ipsilateral notablemente desplazada. Además, la lesión mostraba cambios quísticos, con una extensa presencia de cuerpos psamomatosos muy vascularizados 


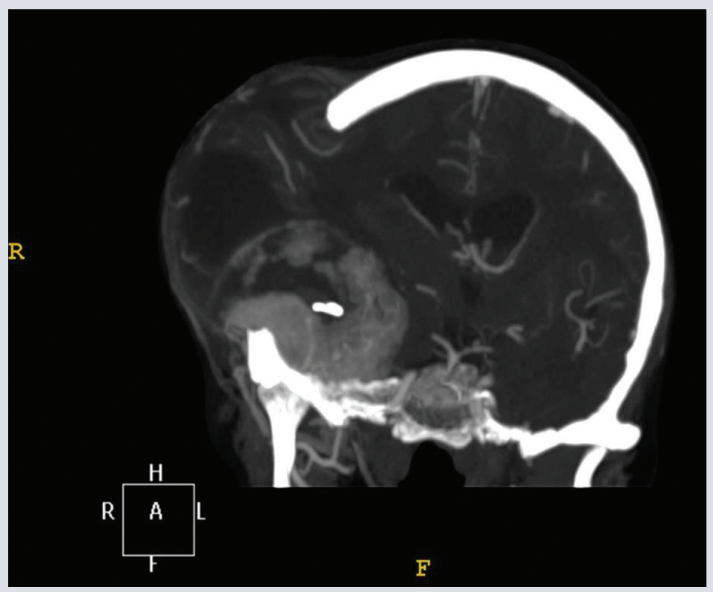

Figura 2. Reconstrucción coronal en algoritmo MIP. Herniación tumoral con compresión sobre territorio de arteria cerebral media derecha.

(Fig. 3). La conclusión del estudio orienta a «descartar variante anaplásica».

La impresión diagnóstica fue: 1) primitivo vs. recidiva, y 2) lesiones vasculares periventriculares.

La paciente fue sometida a tratamiento endovascular mediante embolización prequirúrgica de los sectores aferentes, desde el territorio carotídeo externo, el 8 de marzo de 2019, con lo que se consiguió la oclusión total de los vasos involucrados, con un resultado satisfactorio (Figs. 4 y 5).

\section{DISCUSIÓN}

Usualmente, los meningiomas, en TC y resonancia magnética ( $R M)$ craneales, tienen características morfológicas similares. Típicamente, son tumores redondos, solitarios, que contactan con la duramadre, de

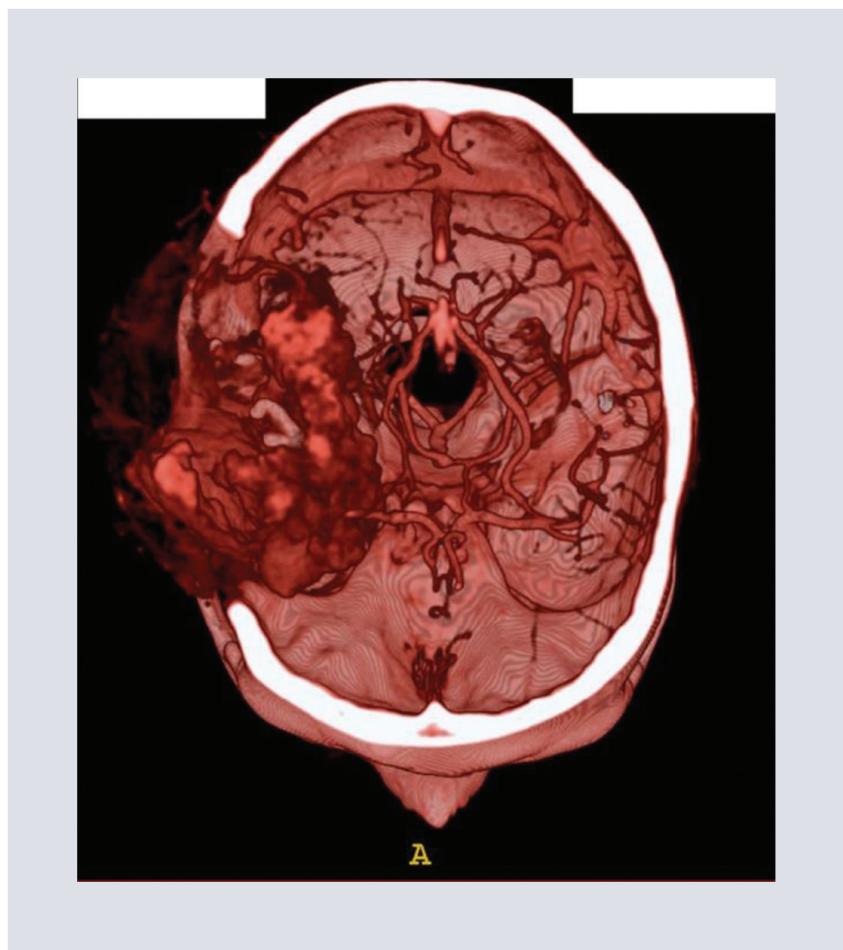

Figura 3. Reconstrucción volumétrica craneocaudal de la tumoración. Herniación tumoral con compresión sobre el territorio de la arteria cerebral media derecha. Nótese los abundantes cuerpos de psamoma.

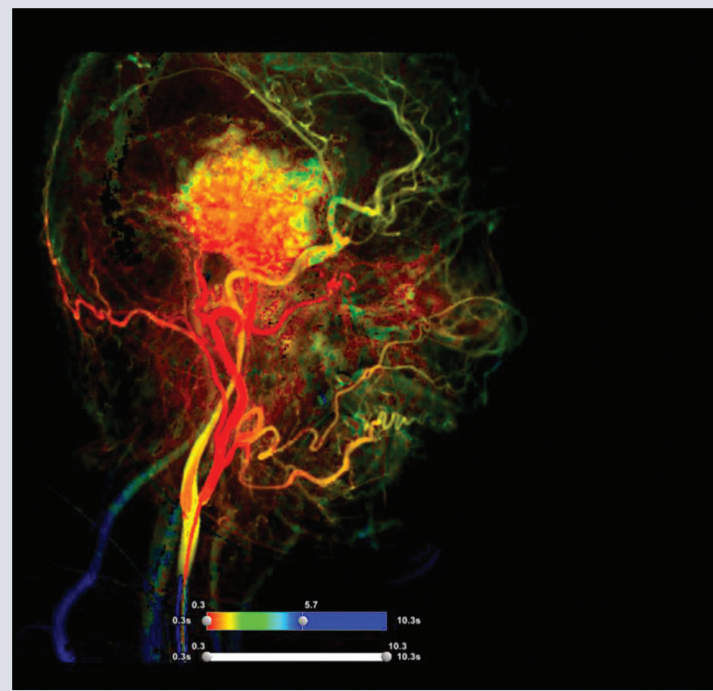

Figura 4. Imagen de perfusión $2 \mathrm{D}$ preembolización. Nótese la intensa vascularización del meningioma por vía de la carótida externa ipsilateral. 


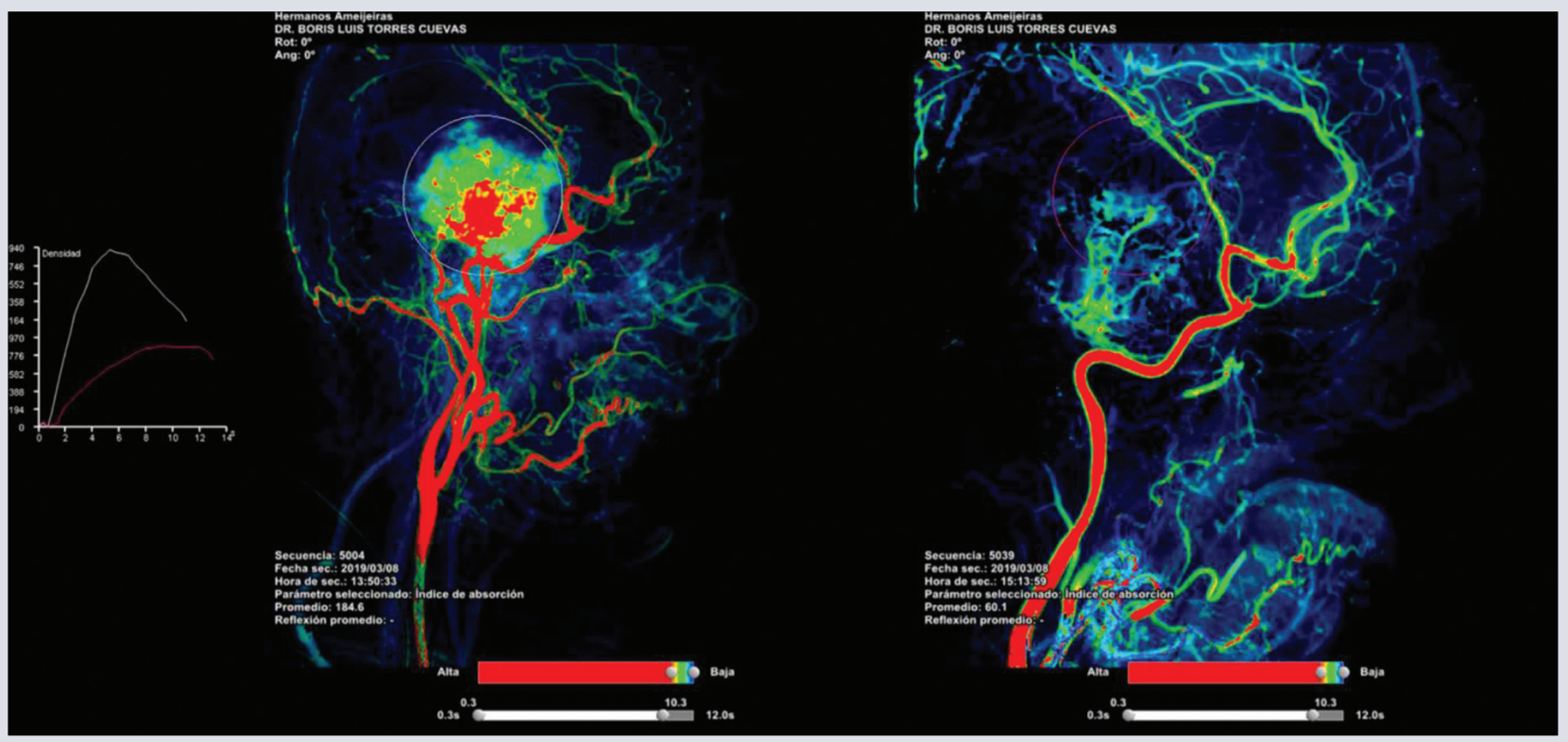

Figura 5. Imagen de perfusión 2D preembolización (izquierda) y después de la embolización (derecha). Nótese la sensible disminución de la vascularización tumoral posterior a la embolización.

bordes bien definidos, con realce intenso y homogéneo después de la administración del contraste, que permite localizarlos con exactitud. En la RM, son isointensos en las imágenes potenciadas en $\mathrm{T} 1$, e isointensos o hiperintensos en las imágenes potenciadas en T2, pero pueden ser heterogéneos si el tumor tiene componente de mayor malignidad, y raramente se calcifican (grado de la OMS II o III) $)^{1,12}$.

Las localizaciones más frecuentes de los meningiomas son la convexidad, región parasagital, ala del esfenoides, espinales, surco olfatorio y fosa media craneal $^{13}$, que fue la encontrada en este caso.

Dependiendo de la localización anatómica, el tamaño y el grado de crecimiento, es frecuente la aparición de cefalea recurrente, con tendencia a la cronicidad, déficits neurológicos y convulsiones ${ }^{14}$. También pueden ocurrir cambios en la personalidad (usualmente confundidos con depresión o demencia) en algunos pacientes con meningiomas de la convexidad o con meningiomas extensos frontobasales ${ }^{5,15}$. En el caso presentado, el cuadro clínico se desarrolla con cambios en el comportamiento y déficits neurológicos, expresados en dificultades motoras y relajación de esfínteres, coincidiendo con lo reportado.

El tratamiento de elección es la cirugía, con la intención de lograr resección macroscópica de dura y hueso; sin embargo, en caso de no garantizarse, se debe dar manejo complementario con radioterapia o quimioterapia. En el caso evaluado, la paciente había sido intervenida con exéresis del tumor. 
Las recomendaciones sugieren realizar seguimiento imagenológico cada 6 meses $^{16}$ para descartar evidencia de crecimiento tumoral, pues la probabilidad de recurrencia es elevada y pueden aparecer metástasis sistémicas ${ }^{1}$. En el caso presentado, la paciente acude 2 meses después de la resección quirúrgica, con sintomatología coincidente con el cuadro clínico del meningioma. Los dos estudios radiológicos realizados (TC craneal simple y angio-TC) apuntan hacia el diagnóstico de meningioma maligno.

El caso que aquí se reporta tiene características coincidentes con la epidemiología (edad alrededor de la media reportada de 60 años, género femenino), aunque en los casos malignos, se reporta mayor frecuencia en el sexo masculino ${ }^{17}$, y sintomatología clínica coincidente con las descritas.

Considerando que menos del 3\% de los nuevos diagnósticos se corresponden con un meningioma maligno de grado $\mathrm{III}^{9}$, es importante describir la evolución de los casos que se diagnostiquen, en aras de contribuir al estado del arte y aportar elementos que permitan mejorar el diagnóstico y el manejo.

\section{CONCLUSIONES}

Un meningioma anaplásico o maligno constituye un caso de rara aparición, por lo que no existe una óptima conducta de tratamiento y la probabilidad de recurrencia es elevada. Se hace difícil establecer criterios para el diagnóstico desde el punto de vista clínico, pues muchos aparecen incidentalmente o son asintomáticos y, en los casos sintomáticos, el cuadro clínico usualmente se corresponde con manifestaciones que pueden ser producidas por diferentes síndromes neurológicos.

El desarrollo de los estudios neurorradiológicos permite realizar el diagnóstico de forma incidental, incluso en los meningiomas de pequeño tamaño y asintomáticos. En el caso evaluado, todos los exámenes radiológicos empleados confluyen hacia el mismo diagnóstico, indicando la sensibilidad de los mismos para el diagnóstico, sobre todo en los meningiomas de alto grado según la clasificación de la OMS.

\section{CONFLICTO DE INTERESES}

Los autores declaran no tener conflicto de interés en relación a este artículo.

\section{FINANCIAMIENTO}

Los autores declaran que este trabajo no requirió de financiamiento.

\section{RESPONSABILIDADES ÉTICAS}

Protección de personas y animales. Los autores declaran que para esta investigación no se han realizado experimentos con seres humanos ni en animales.

Confidencialidad de los datos. Los autores declaran que han seguido los protocolos de su centro de trabajo y en este artículo no aparecen datos de pacientes.

Derechos a la privacidad y consentimiento informado. Los autores han obtenido el consentimiento informado de los pacientes $\mathrm{y} / \mathrm{o}$ 


\section{sujetos referidos en el artículo. Este documento obra en poder del autor de correspondencia.}

\section{BIBLIOGRAFÍA}

1. Gómez-Vega JC, Ocampo Navia MI, Feo Lee O. Epidemiología y caracterización general de los tumores cerebrales primarios en el adulto. Univ Med. 2019;60(1):1-14

2. Cushing $H$. The meningiomas (dural endotheliomas): their source, and favoured seats of origin. Brain. 1992;45:282-316.

3. Perry A, Louis DN, Scheithauer BW, et al. Meningiomas. En: Louis DN, Ohgaki H, Wiestler OD, Cavenee WK, ed. WHO classification of tumors of the central nervous system. $4 .^{a}$ ed. Lyon: International Agency for Research on Cancer (IARC), Press; 2007. p. 164-72.

4. Fathi AR, Roelcke U. Meningioma. Curr Neurol Neurosci Rep. 2013;13(4):337.

5. Casas PI, Baez A, Banfi N, Blumenkrantz Y, Halfon MJ, Barros M, et.al. Meningiomas en neurooncología. Neurología Argentina. 2016; 8(3):210-26.

6. Miranda-Maldonado IC, LarraldeContreras L, Niderhauser-García A, Barboza-Quintana O, AncerRodríguez J. Clasificación morfológica de meningiomas en una casuística del Hospital Universitario Dr. José E González. Patología Rev Latinoam. 2011;49(3):188-95.
7. Wiemels J, Wrensch M, Claus EB. Epidemiology and etiology of meningioma. J Neurooncol. 2010;99:307-14.

8. Perry A, Scheithauer BW, Stafford SL, Lohse CM, Wollan PC. «Malignancy» in meningiomas: a clinicopathologic study of 116 patients. Cancer. 1999;85:2046-56.

9. Rogers L, Barani I, Chamberlain M, Kaley T, McDermott M, Raizer J, et. al. Meningiomas: Knowledge Base, Treatment Outcomes, and Uncertainties: A RANO Review. J Neurosurg. 2015;122(1):4-23.

10. Durand A, Labrousse F, Jouvet A, Bauchet L, Kalamarides M, Menei P, et al. WHO grade II and III meningiomas: a study of prognostic factors. J Neurooncol. 2009;95:367-75.

11. Le May DR, Bucci MN, Farhat SM. Malignant transformation of recurrent meningioma with pulmonary metastases. Surg Neurol. 1989;31:365-8.

12. Aguilar M, Salvático R. Signo de la hoja de malvón y meningiomas en resonancia magnética. RAR. 2012;76(1):1-3.

13. Hallinan TPD, Hegde AN, Lim WEH. Dilemmas and diagnostic difficulties in meningioma. Clin Radiol. 2013;68:837-44.

14. McDermott MW, Wilson CB. Meningeomas. Neurological Surgery 1997;4:127- 59

15. García-Navarrete E, Sola RG. Aspectos clínicos y quirúrgicos de los meningiomas de la base del cráneo. Rev Neurol. 2002;34:584-92.

16. Lopez Flores G, Samblas Garcia J, Guatierrez-Diaz JA, Bustos P de Salcedo JC, Sallanbanda Diaz K. Historia natural y clasificaciones de los meningiomas de la base craneal. Rev Mex de Neuroci. 2011;12 (1):38-49.

17. NIH. National Cancer Institute and Center for Cancer Research. Meningioma. 2017. Disponible en: https://www.cancer.gov/nci/rare-brain-spinetumor/tumors/meningioma. 